\title{
Adaptación al Español de la Escala de Autoeficacia General para su Uso en el Contexto Ecuatoriano
}

\author{
Spanish Adaptation of the General Self-Efficacy Scale for Use in Ecuadorian Context
}

\author{
Alexandra Bueno-Pacheco ${ }^{1}$, Sandra Lima-Castro ${ }^{2}$, Eva Peña-Contreras ${ }^{3}$, Cristina Cedillo-Quizhpe ${ }^{4}$ \\ y Mónica Aguilar-Sizer ${ }^{5}$
}

\section{Resumen}

La autoeficacia tiene amplia relevancia en el ámbito de la psicología clínica y de la salud, puesto que puede explicar un amplio rango de comportamientos de las personas, ya que se refiere a la percepción de la capacidad que poseen para alcanzar un objetivo. En el presente estudio se plantearon dos objetivos: la adaptación al español dela Escala de Autoeficacia General (EAG)para su uso en contexto ecuatoriano y la evaluación de la reproducción de la estructura factorial propuesta por sus autores,en una muestra de 567 participantes. Se analizó la confiabilidad, dimensionalidad y validez de la escala.Los resultados muestran una adecuada consistencia interna, excelentes índices de discriminación de cada uno de los ítems, altas cargas factoriales y además una validez convergente y discriminante frente al constructo de Bienestar y de emociones negativas (Modelo PERMA). Con ello se concluye que los datos se ajustan al modelo teórico, obteniendo un instrumento útil para los profesionales de la salud en el contexto ecuatoriano.

Palabras clave: Escala de Autoeficacia General, propiedades psicométricas, análisis de ítems, adaptación de test, bienestar

\begin{abstract}
Self-efficacy has great relevance in clinical and health psychology, since it can explain a wide range of behaviors, because it refers to the beliefs people have about their own capabilities to reach a goal. For the purpose of this study, two objectives were proposed: the Spanish adaptation of the General Self-Efficacy Scale (GSE) created by Schwarzer and Jerusalem (1995) in order to be used in the ecuadorian context and the evaluation of the reproduction of the factorial structure proposed by its authors, considering a sample of 567 participants. The reliability, dimensionality and validity of the scale were analyzed. The results show an adequate internal consistency, excellent discrimination indices for each item, high factor loads and also a convergent and discriminant validity against the Wellbeing and negative emotions constructs (PERMA). We may conclude that the data adjust well the theoretical model, obtaining a useful tool for health professionals in the ecuadorian context.
\end{abstract}

Keywords: General Self-Efficacy Scale, psychometrics properties, item analysis, adaptation test, wellbeing

\footnotetext{
${ }^{1}$ Magíster en Diagnóstico y Rehabilitación Neuropsicológica. Docente de la Universidad de Cuenca. Universidad de Cuenca, Facultad de Psicología. Av. 12 de Abril y Agustín Cueva. Apartado: 01.01.168, Ecuador. Tel.: +593991506998. Correo: alexandra.bueno@ucuenca.edu.ec

${ }^{2}$ Magíster en Psicoterapia Integrativa y Psicología de la Salud, Doctoranda en Neurociencias y Psicología Clínica. Directora del proyecto "Variables asociadas al bienestar en personas adultas con y sin discapacidad". Docente de la Universidad de Cuenca, Ecuador.

${ }^{3}$ Magíster en Psicoterapia Integrativa. Docente de la Universidad de Cuenca, Ecuador.

${ }_{5}^{4}$ Magíster en Educación y Desarrollo del Pensamiento. Docente de la Universidad de Cuenca, Ecuador.

${ }^{5}$ Magíster en Intervención, Asesoría y Terapia Familiar Sistémica. Docente de la Universidad de Cuenca, Ecuador.
} 


\section{Introducción}

La autoeficacia, en palabras de su máximo exponente, Bandura (1977) hace referencia a la creencia que tienen las personas acerca de sus propias habilidades para llevar a cabo conductas adecuadas, de manera que les permita alcanzar un objetivo de forma exitosa.

Otros autores afirman que dichas creencias dependerán de sus expectativas acerca de las aptitudes que poseen así como de la valoración que se haga de las dificultades asociadas a la tarea objetivo (Acuña-Gurrola \& González-CelisRangel, 2010). Esta propuesta resulta interesante en tanto Bandura (1997) distingue dos situaciones: la expectativa de resultado y la expectativa de eficacia. La primera se refiere a la valoración de que con cierto comportamiento se obtendrá un resultado;por el contrario, la expectativa de eficacia implica la convicción de que la persona, en efecto, puede realizar cierta conducta que lleve a un resultado (Bandura, 1997).

Siguiendo esta línea se comprende que el resultado que la persona espera de un acto, no influye en su percepción de la capacidad que tiene para realizar las acciones (Williams \& Rhodes, 2016); y en cambio resalta la injerencia de la autoeficacia en el comportamiento de las personas, pues regula y brinda la motivación para que se ejecuten una serie de actuaciones en pos de un objetivo (Bandura, 2006).

La autoeficacia por ende, se convierte en un factor crucial en la conducta de las personas, pues la concepción de sus competencias determinará el rumbo de sus acciones hacia diferentes metas (Singh, Shukla, \& Singh, 2010). Por ello se considera un constructo con estilo prospectivo, ya que promueve el desempeño de tareas que demandan más esfuerzo y que se dirigen hacia metas más altas, en las que a su vez,se invierte mayor cantidad de tiempo yrequieren ser más persistentes (Brenlla, Aranguren, Rossaro, \& Vázquez, 2010; Reyes, Montenegro, Knoll, \& Schwarzer, 2014; Schwarzer \& Warner, 2013). En contraparte, se ha evidenciado que aquellas personas que creen que no tienen poder para controlar los sucesos de su vida, perderán el interés para actuar y afrontar las dificultades, y finalmente no harán ningún intento (Bandura, 1977).
Si bien la autoeficacia se proyecta como un factor de dominio específico, pudiendo interpretarse un mayor o menor nivel de autoeficacia ante diversas situaciones, también puede ser considerada en un sentido general para identificar la confianza global o el juicio generalizado que las personas poseen para afrontar situaciones novedosas o estresantes; es decir, cuán eficientes son en varios aspectos (Luszczynska, Scholz, \& Schwarzer 2005; Schwarzer, Bäßler, Kwiatek, Schröder, \& Zhang, 1997).

De esta manera, al ser considerada la autoeficaciacomo el principal factor que influye en la conducta, podría además explicar un amplio rango del comportamiento de las personas, sobre todo al estudiar varias conductas de manera simultánea y las conductas de afrontamiento (Luszczynska, Scholz et al., 2005).

Considerando la importancia de este constructo, se creó la Escala de Autoeficacia Generalizada (General Self-Efficacy Scale; Schwarzer \& Jerusalem, 1995); cuya versión original en idioma Alemán pertenece a Matthias Jerusalem y Ralf Schwarzer en el año 1979 (Schwarzer, 2014). Esta escala, que ha sido traducida al español (Bäßler \& Schwarzer, 1996) y alrededor de 30 idiomas gracias a otros coautores (Schwarzer, 2014), fue creada con el objetivo de evaluar la percepción de la autoeficacia general de personas,mediante la cual puedan adaptarse y afrontar los problemas de la vida diaria así como situaciones estresantes de todo tipo (Schwarzer \& Jerusalem, 1995).

Los autores señalan que la prueba tiene una consistencia interna buena $(\alpha>8$; Schwarzer, 2014) y varios estudios a nivel de latinoamérica lo avalancon valores de consistencia interna similares o inferiores (Brenlla et al., 2010; Cid, Orellana, \& Barriga, 2010; Grimaldo, 2005; Padilla, Acosta, Gómez, Guevara, \& González, 2006).

Con esta escala se han realizado comparaciones multiculturales entre varios países, lo que avala de manera empírica la universalidad y unidimensionalidad del constructo de autoeficacia (Brenlla et al., 2010). Se citan por ejemplo los estudios realizados entre Alemania, Polonia y Corea del Sur (Luszczynska, Scholz et al., 2005) o aquellos realizados entre Costa Rica, 
Alemania, Polonia, Turquía y Estados Unidos (Luszczynska, Gutiérrez-Doña, \& Schwarzer, 2005). Dichas investigaciones además, han ayudado a determinar la validez de criterio al relacionar la autoeficacia general con varios constructos como la autopercepción positiva (Molina, Raimundi, López, Cataldi, \& Bugallo, 2011), la resiliencia (Schwarzer \& Warner, 2013) y el bienestar que manifiestan las personas (KleinHessling, Lohaus, \& Ball, 2005; González, Valle, Freire, \& Ferradas, 2012). Este último constructo resulta muy importante puesto que se considera una meta común y universal de las personas (Aguado et al, 2012).

El bien estar se comprende de forma multidimensional (Huppert \& So, 2013) puesto que implica que la persona se sienta bien y tenga un funcionamiento óptimo para afrontar los desafíos de la vida; para ello, Seligman (2011) considera cinco dimensiones que engloban el bienestar: emociones positivas, compromiso, relaciones personales, significado de vida y logro. Los constructos de bienestar y autoeficacia han sido relacionados positivamente puesto que implica que la persona tenga una percepción adecuada de sus capacidades y pueda desempeñarse de forma satisfactoria (González et al, 2012; Ortega \& Cuadrado, 2011; Singh et al, 2010).

Así mismo, varios autores determinan que existe una relación directa y positiva entre la autoeficacia y algunas pautas de comportamiento, como la práctica de conductas saludables (Chavarria, Stevens, Jason, \& Ferrari, 2012; Luszczynska \& Schwarzer, 2005; del Castillo, Guzmán, Iglesias, \& Reyes, 2012),el control de enfermedades o manejo del dolor (del Castillo et al., 2012; Nickel, Spink, Andersen, \& Knox, 2014; Olivari \& Urra, 2007; Schüz, Wurm, Warner, \& Ziegelmann, 2012; Tewary \& Farber, 2012), el establecimiento de relaciones amorosas íntimas (Riggio et al., 2013) y la práctica de actividad física a largo plazo (Lewis, Williams, Frayeh, \& Marcus, 2016; Reyes et al., 2014; Ryan, 2008). De manera opuesta, se han realizado estudios que involucran a varios países del continente americano y europeo, que señalan que la autoeficacia correlaciona de manera negativa con estrés, depresión y ansiedad (Luszczynska, Gutiérrez-Doña, \& Schwarzer, 2005; Olivari \&
Urra, 2007).

Tal como se ha presentado, es de vital importancia la percepción de la autoeficacia en el establecimiento de los logros y la consecución de los mismos, así como en la capacidad y forma de afrontar las situaciones cotidianas, ya sea a nivel personal o con repercusiones a nivel social.

Sin embargo, a pesar de la clara importancia de esta variable, los estudios desarrollados para evaluarla en el contexto latinoamericano aún son escasos (Reyes-Jarquín \& Hernández-Pozo, 2011) y nuestra investigación ratifica que, de manera específica a nivel nacional, son inexistentes; por eso resulta imprescindible contar con instrumentos en el ámbito de la salud que evalúen esta variable, con el objeto de fomentar el autoconocimiento de las personas para apoyar su actuación frente a situaciones estresantes, o relacionadas con la consecución de metas y su bienestar general. Así mismo, una herramienta de esta naturaleza serviría como instrumento de soporte en las intervenciones de personas de grupos vulnerables, como aquellas con discapacidad o adultos mayores, con el fin de manejar estrategias de afrontamiento adecuadas ante diversas situacionesque amenazan su estabilidad física y emocional.

Es por ello que este trabajo se plantea un doble propósito, por una parte adaptar la Escala de Autoeficacia Generalizada para su uso en Ecuador y por otra parte evaluar la reproducción de la estructura factorial propuesta por sus autores. Con esto lograríamos brindar mejores herramientas para el uso en el ámbito de la salud en nuestro país, siendo los psicólogos los más beneficiados con ello (Martorell \& Gómez, 2010). Además de ello, se espera evaluar la correlación de esta escala conotros constructos como el bienestar percibido $\mathrm{y}$ emociones negativas como enojo, angustia o tristeza.

\section{Método}

La adaptación de test psicométricos, dentro de los cuales podemos considerar este estudio, sigue procesos rigurosos y claramente definidos por la International Test Commission (2016), que señala las directrices para la traducción y adaptación de test, los cuales han sido trabajados en lengua española por Muñiz, Elosua y Hambleton (2013). 
En consecuencia, el presente trabajo se define como un estudio cuantitativo, centrado en un análisis descriptivo, correlacional-causal de los ítems del test; que utiliza los procedimientos y técnicas estadísticas clásicas para estudios psicométricos, como lo son el análisis de ítems bajo la teoría clásica y el análisis factorial en sus versiones exploratoria y confirmatoria.

\section{Participantes}

Inicialmente se realizó una prueba piloto de 121 personas con la finalidad de abordar los procesos iniciales de validez y confiabilidad (40.5\% hombres y $59,5 \%$ mujeres) con edad media de 44 años.

La muestra definitiva del estudio estuvo integrada por 567 participantes, con un margen de error de $4.1 \%$ frente a un universo de $8,766,811$ hombres y mujeres de entre 19 y 89 años de edad en Ecuador continental(Instituto Nacional de Estadísticas y Censos [INEC], 2010) en base al 95\% de confiabilidad. La edad media (M) del grupo general fue de 49.07 años con una desviación estándar (DE) de 18.2 años. De la muestra, 267 son varones y equivalen al $48.7 \%$ $(M=46.94 ; \quad D E=18.12)$ y 291 mujeres que representan el 51.3\% ( $M=51.09 ; D E=18.07)$. Un total de $49.6 \%$ de la muestra eran personas casadas, mientras que el $23.6 \%$ era soltero; el $21.3 \%$ de las mujeres eran Amas de Casa. El $55.9 \%$ del grupo tiene trabajo y sólo el $42.7 \%$ manifestó tener estabilidad laboral. El 20.1\% del grupo manifestó padecer discapacidad física, congénita o adquirida. Con esta caracterización heterogénea de la muestra aseguramos contar con diversidad de sujetos para que la misma sea representativa de la población. A los participantes se los contactó en sus lugares de trabajo y en centros de atención al público en general.

\section{Instrumentos}

General Self-Efficacy Scale (Schwarzer \& Jerusalem, 1995). La escala está integrada por 10 ítems que se valoran através de una escala Likert de cuatro puntos. En muestras de 23 naciones se ha registrado un alfa de Cronbach $(\alpha)$ entre .76 y .9 y se ha confirmado una estructura unidimensional (Schwarzer, 2014); para su adaptación a la población ecuatoriana se usó el método traducción - retrotraducción (back translation). La versión en inglés de Schwarzery Jerusalem (1995) fue sometida a un proceso de traducción y retrotraducción por parte de una psicóloga de origen canadiense y luego fue analizada por los miembros del equipo investigador para comprobar el grado en que los ítems de la escala representan el concepto de autoeficacia y se ajustan al argot cultural ecuatoriano. Aunque las variaciones sugeridas de la versión traducida son pocas, en comparación con otras versiones en español, es importante destacar que de acuerdo con la opinión de los expertos, estas frases añadidas facilitan la comprensión del reactivo por parte del respondiente.

Esta primera versión se probó en la prueba piloto aplicada a 121 personas, y no se evidenciaron mayores cambios en comparación con la escala original;sin embargo, la traducción de las etiquetas de la escala Likert que fueron: $1=$ Completamente falso, $2=$ Algunas veces falso, $3=$ Alguna vecescierto, y $4=$ Completamente cierto, reportaron algunos problemas de comprensión. Posteriormente, con esta información se concibió la versión final donde las etiquetas Likert fueron modificadas para su fácil comprensión, según se señala: $1=$ Nunca, $2=$ Pocas veces, $3=$ Muchas veces, $4=$ Siempre.

Con el fin de abordar otros aspectos relacionados con la validez del constructo, particularmente con la validez convergente $\mathrm{y}$ discriminante; se consideraron otras escalas relacionadas, como se expresan a continuación:

PERMA-Profiler (Butler \& Kern, 2016). La escala mide los cinco pilares del Bienestar definidos por Seligman (2012): emociones positivas, compromiso, relaciones, significado $\mathrm{y}$ logros. Evalúa también tres factores independientes como salud, emociones negativas y soledad. La escala está compuesta por 23 ítems en total; de ellos, 15 evalúan los cinco pilares del bienestar (3 ítems para cada factor), más uno que representa "bienestar general" (ítem criterial), los demás ítems evalúan los factores independientes. Cada ítem se califica en una escala Likert, que va de 0 (nunca) a 10 (siempre). Para el presente estudio, se consideraron únicamente los ítems relacionados con los pilares del bienestar y el factor independiente referido a emociones 
negativas, de la adaptación ecuatoriana de esta escala (Lima-Castro, Peña-Contreras, CedilloQuizhpe, \& Cabrera-Vélez, 2016).

Adicionalmente se aplicó un cuestionario demográfico con el fin de apoyar la caracterización de la muestra en cuanto a la edad, nivel educativo, nivel de ingresos económicos, situación laboral y presencia de discapacidad, con el fin de facilitar los criterios comparativos en los análisis posteriores.

\section{Procedimiento}

La aplicación de las pruebas tuvo una duración aproximada de 20 minutos, y se llevó a cabo en instituciones laborales o centros públicos y privados de atención en general de la ciudad de Cuenca, durante el transcurso del año 2016. La participación de los sujetos fue voluntaria y anónima; para ello previamente firmaron una carta de consentimiento informado, en la cual se explicaban los fines investigativos de la evaluación y la confidencialidad de los datos obtenidos.

Se controló el efecto del orden de presentación de las escalas utilizadas de manera balanceada, aplicando el cuestionario de variables sociodemográficas al final del instrumento.

\section{Análisis de Datos}

A partir de la prueba piloto se realizó un análisis clásico de los ítems basado en el estudio de la validez de contenido, índices de discriminación, dimensionalidad y sensibilidad cultural (Cohen \& Swerdlik, 2006). El índice de correlación ítem - total y el método usado para evaluar la confiabilidad de la escala fue el coeficiente Alfa de Cronbach ( $\alpha$ ). En esta misma fase se aplicó un Análisis Factorial Exploratorio (AFE), con el fin de evaluar la dimensionalidad del constructo por medio del método de mínimos cuadrados generalizados; estos análisis se realizaron con el programa SPSS Versión 23.0.

Con la muestra definitiva se realizaron dos análisis. El primer paso consistió en evaluar la estructura factorial de la escala una vez que se realizaron los breves cambios derivados de la prueba piloto. El Análisis Factorial Exploratorio (AFE) se realizó con el software FACTOR 7.0 (Lorenzo-Seva \& Ferrando, 2006). Posteriormente se aplicó un Análisis Factorial Confirmatorio
(AFC) que permitiese ratificar la estructura unifactorial de la escala, para estimar la confiabilidad del instrumento y evaluar el ajuste de los datos al modelo teórico planteado.

En la fase de estimación de parámetros del AFC se adoptó como método el procedimiento robusto de máxima verosimilitud (ML, Robust). Durante la fase confirmatoria se utilizó software que permitiera trabajar con datos de entrada en forma de matrices de correlaciones policóricas de los ítems, como el FACTOR 7.0 (Lorenzo-Seva \& Ferrando, 2006) y el Lisrel 8.8 (Jöreskog \& Sörbom, 2006).

En la fase de evaluación de la bondad del ajuste del modelo, se consideran los índices clásicos como el chi cuadrado de Satorra-Bentler, que corrige el efecto de la violación de la distribución normal de los datos; el chi-cuadrado relativo, referido al cociente entre el valor del chicuadrado y los grados de libertad; el error cuadrático medio de aproximación (RMSEA), el índice de ajuste comparativo (CFI) y la raíz media cuadrática residual (RMSR). Para efectos de interpretación se toma como referente los valores sugeridos por Hu y Bentler (1999), en los que el ajuste se considera adecuado cuando el valor de chi cuadrado es bajo, el valor de RMSEA no excede de .06, CFI es mayor que .95 y RMSR es inferior a .08 .

Adicionalmente, para potenciar los criterios de validez del constructo (convergente $y$ discriminante), se realizó un análisis de las correlaciones entre las puntuaciones de la escala de Autoeficacia con las puntuaciones de la dimensión de Bienestar y de las emociones negativas, aplicando las recomendaciones de Campbell y Fiske (1959), que indican que existe validez convergente entre dos constructos sito das las correlaciones entre los indicadores son significativas, criterio adoptado también para evaluar la validez discriminante.

\section{Resultados}

\section{Pilotaje}

Los resultados de la primera fase, referida a la prueba piloto, fueron satisfactorios; respecto al análisis de los ítems, todos ellos mostraron un índice de discriminación superior a .65 . La 
Tabla 1. Índices de Discriminación (ID), Media $(\bar{X})$ y Desviación Estándar (DE) de los ítems de la EAG

\begin{tabular}{lccc} 
Item & ID & $\bar{X}$ & $D E$ \\
\hline $\begin{array}{l}\text { 1.A pesar de los obstáculos yo puedo encontrar las } \\
\text { maneras de obtener lo que quiero }\end{array}$ & .52 & 3.14 & .710 \\
$\begin{array}{l}\text { 2. Yo enfrento problemas graves si me esfuerzo lo } \\
\text { suficiente }\end{array}$ & .43 & 3.05 & .817 \\
$\begin{array}{l}\text { 3. Es fácil mantenerme en mis metas hasta } \\
\text { alcanzarlas }\end{array}$ & .50 & 3.13 & .766 \\
$\begin{array}{l}\text { 4. Me siento seguro de poder enfrentar eficazmente } \\
\text { situaciones inesperadas }\end{array}$ & .63 & 3.13 & .827 \\
$\begin{array}{l}\text { 5. Gracias a mis cualidades personales yo puedo } \\
\text { enfrentar situaciones inesperadas }\end{array}$ & .70 & 3.20 & .760 \\
$\begin{array}{l}\text { 6. Yo puedo mantener la calma cuando estoy en } \\
\text { problemas porque confío en mis habilidades para }\end{array}$ & .59 & 3.06 & .826 \\
$\begin{array}{l}\text { enfrentarlos } \\
\begin{array}{l}\text { 7. Venga lo que venga, por lo general soy capaz de } \\
\text { manejarlo }\end{array}\end{array}$ & .67 & 3.25 & .744 \\
$\begin{array}{l}\text { 8. Yo puedo resolver la mayoría de los problemas } \\
\text { si me esfuerzo lo necesario }\end{array}$ & .62 & 3.35 & .671 \\
$\begin{array}{l}\text { 9. En una situación difícil, generalmente se me } \\
\text { ocurre lo que debo hacer }\end{array}$ & .60 & 3.09 & .784 \\
$\begin{array}{l}\text { 10. Cuando me enfrento a un problema, } \\
\text { generalmente encuentro varias soluciones }\end{array}$ & .59 & 3.17 & .750 \\
\hline
\end{tabular}

Tabla 2. Correlaciones entre la escala de Autoeficacia General y las dimensiones de Bienestar y de Emociones Negativas de la Escala PERMA

\begin{tabular}{lcccccc}
\hline & Logro & $\begin{array}{c}\text { Emociones } \\
\text { Positivas }\end{array}$ & Compromiso & Significado & $\begin{array}{c}\text { Relaciones } \\
\text { Positivas }\end{array}$ & $\begin{array}{c}\text { Emociones } \\
\text { Negativas }\end{array}$ \\
\hline Autoeficacia General & $.336^{* *}$ & $.281^{* *}$ & $.273^{* *}$ & $.416^{* *}$ & $.246^{* * *}$ & $-.159^{* * *}$ \\
Logro & & $.484^{* *}$ & $.411^{* *}$ & $.621^{* *}$ & $.447^{* *}$ & $-.123^{* *}$ \\
Emociones Positivas & & & $.298^{* *}$ & $.514^{* * *}$ & $.432^{* * *}$ & $-.262^{* * *}$ \\
Compromiso & & & & $.443^{* *}$ & $.312^{* *}$ & -.014 \\
Significado & & & & & $.477^{* *}$ & $-.140^{* *}$ \\
Relaciones Positivas & & & & & & $-.150^{* *}$ \\
\hline
\end{tabular}

**. La correlación es significativa al nivel .01 (bilateral).

confiabilidad, estimada mediante el coeficiente $\alpha$ de Cronbach, fue de .91, el cual se reduciría en caso de eliminar algún elemento. Con el AFE se obtuvo una estructura unifactorial; los supuestos de adecuación de la muestra y otros valores resultaron óptimos $(\mathrm{KMO}=.92$; Valor del determinante $=.03$; Porcentaje de varianza explicada $=53 \%$ ). Durante el proceso de administración y registro de la información se confirmó, una vez aplicada la versión final traducida al español y adaptada al contexto ecuatoriano, que ninguno de los participantes mostrara algún problema de comprensión de los ítems. De este modo se garantiza la intencionalidad de la medida del ítem y la claridad de su redacción, elementos fundamentales de la validez del contenido.

\section{Capacidad de discriminación de los ítems}

Una vez comprobadas las propiedades psicométricas de la escala y explorada su estructura unifactorial, se analizaron sus propiedades psicométricas en la muestra definitiva; para ello y en primer lugar, se procedió a realizar un análisis de los ítems bajo el enfoque clásico, con la finalidad de valorar su capacidad discriminativa. Los resultados arrojados evidencian excelentes índices de discriminación, superiores a .40 en todos los casos, por lo que no fue necesario eliminar ningún ítem; además, se calculó la media y desviación típica de cada ítem (Tabla 1).

\section{Validez convergente y discriminante}

En la Tabla 2 se muestran las correlaciones entre la escala de Autoeficacia General frente a las 
Tabla 3. Propiedades psicométricas de la escala PERMA

\begin{tabular}{lcccccc}
\hline Subescala & $\begin{array}{c}\text { Correlación } \\
\text { interítem } \\
\text { promedio }\end{array}$ & Alfa & Media & $\begin{array}{c}\text { Desviación } \\
\text { Estándar }\end{array}$ & Mín & Máx \\
\hline Emociones Positivas & .4564 & .7158 & 8.173734 & 1.373384 & 1 & 10 \\
Compromiso & .3633 & .6313 & 8.413428 & 1.256748 & 4 & 10 \\
Relaciones Positivas & .3834 & .651 & 7.935332 & 1.554952 & 2 & 10 \\
Significado & .4606 & .7192 & 8.466784 & 1.318239 & 2.67 & 10 \\
Logro & .341 & .6082 & 7.864785 & 1.334856 & 3.33 & 10 \\
Emociones Negativas & .5669 & .797 & 4.49853 & 2.073433 & 0 & 10 \\
PERMA* & .3542 & .8916 & 8.168968 & 1.100643 & 4.2 & 10 \\
\hline *VRM
\end{tabular}

*Valoración de los cinco pilares del bienestar en conjunto

Tabla 4. Matriz de Correlaciones policóricas y evaluación de supuestos bajo AFE (*)

\begin{tabular}{lllllllllll}
\hline & I1 & I2 & I3 & I4 & I5 & I6 & I7 & I8 & I9 & I10 \\
\hline I1 & 1 & & & & & & & & & \\
I2 & .41 & 1 & & & & & & & & \\
I3 & .41 & .359 & 1 & & & & & & & \\
$\mathrm{I} 4$ & .43 & .331 & .417 & 1 & & & & & & \\
$\mathrm{I} 5$ & .42 & .429 & .458 & .672 & 1 & & & & & \\
$\mathrm{I} 6$ & .43 & .293 & .318 & .507 & .554 & 1 & & & & \\
$\mathrm{I} 7$ & .46 & .347 & .428 & .543 & .592 & .664 & 1 & & & \\
$\mathrm{I} 8$ & .44 & .417 & .448 & .494 & .530 & .458 & .573 & 1 & & \\
$\mathrm{I} 9$ & .35 & .324 & .397 & .497 & .520 & .444 & .526 & .527 & 1 & \\
$\mathrm{I} 10$ & .37 & .317 & .417 & .445 & .522 & .432 & .501 & .537 & .574 & 1 \\
\hline
\end{tabular}

(*) Determinante de la matriz $=.029$; Estadístico de Bartlett $=1986(g l=45 ; p=0.000010$ ); Test KMO =.90742 (Muy bueno) y BC Bootstrap $95 \%$ intervalo de confidencia de $\mathrm{KMO}=(.897 ; .923)$

Tabla 5. Proporción de varianza explicada, cargas factoriales y valores de comunalidad

\begin{tabular}{lccccc}
\hline Item & Autovalor & $\begin{array}{c}\text { Proporción } \\
\text { de Varianza } \\
\text { Explicada }\end{array}$ & $\begin{array}{c}\text { Proporción } \\
\text { Acumulada }\end{array}$ & $\begin{array}{c}\text { Carga } \\
\text { Factorial }\end{array}$ & Comunalidad \\
\hline I1 & 5.14951 & .51495 & .51495 & .597 & .356 \\
I2 & .87184 & .08718 & & .513 & .263 \\
I3 & .7433 & .07433 & & .588 & .346 \\
I4 & .62619 & .06262 & & .721 & .520 \\
I5 & .60639 & .06064 & & .787 & .619 \\
I6 & .51133 & .05113 & & .681 & .464 \\
I7 & .45525 & .04552 & & .776 & .602 \\
I8 & .42759 & .04276 & & .730 & .533 \\
I9 & .30933 & .03093 & & .687 & .472 \\
I10 & .29928 & .02993 & & .678 & .460 \\
\hline
\end{tabular}

5 dimensiones de Bienestar y la dimensión de Emociones Negativas del PERMA. La validez convergente se determinó por las correlaciones significativas y positivas entre la percepción de autoeficacia y las distintas dimensiones de Bienestar que tienen las personas, sobre todo en los dominios de Logro $(r=.336)$ y Significado $(r=.416)$; por el contrario, la validez discriminante se evidenció con la correlación baja y negativa entre Autoeficacia y emociones negativas de las personas $(r=-.159)$. Las propiedades psicométricas de la escala PERMA para esta muestra se detallan en la Tabla 3.

\section{Dimensionalidad}

Se probó la unidimensionalidad de la escala mediante un AFC, considerando como datos de entrada la matriz de correlaciones policóricas. El 
Tabla 6. Índices de Ajuste

\begin{tabular}{lcc}
\hline Índices de Ajuste & Valor & IC Bootstrap 95\% \\
\hline Root Mean Square Error of Approximation (RMSEA) & .058 & $.0414-.0641$ \\
Fit Function Chi Square with35 degrees of freedom & $56.551(p=0.012028)$ & \\
Chi cuadrado relativo Chi Square/df (56.551/35) & $1.61(<3)$ & \\
Non-Normed Fit Index (NNFI; Tucker \& Lewis) & 0.984 & $.979-.993$ \\
Comparative Fit Index (CFI) & 0.988 & $.984-.994$ \\
Schwarz's Bayesian Information Criterion (BIC) & 229.413 & $195.822-243.247$ \\
Goodness of Fit Index (GFI) & .993 & $.992-.996$ \\
Adjusted Goodness of Fit Index (AGFI) & .991 & $.989-.994$ \\
Goodness of Fit Index without diagonal values (GFI) & .99 & $.987-.994$ \\
Goodness of Fit Index without diagonal values (AGFI) & .987 & $.983-.992$
\end{tabular}

AFE basado en esta matriz permitió validar los supuestos de aplicación (KMO, Barlett y valor del determinante) en forma satisfactoria (Tabla 4). La estructura de un factor fue sometida al AFC utilizando el método de máxima verosimilitud robusto. La solución unifactorial es capaz de explicar en conjunto el $51.49 \%$ de la varianza total con cargas factoriales que oscilan entre $.51 \mathrm{y}$ .78 (Tabla 5); la confiabilidad de la versión adaptada, estimada mediante el coeficiente $\alpha$ de Cronbach, fue de .87 .

\section{Evaluación del Ajuste de la escala}

Para evaluar el ajuste del modelo, se obtuvo un conjunto de índices de ajuste satisfactorios; destacando como principales: el chi-cuadrado relativo $\left(X^{2} / \mathrm{gl}\right)=1.61 ; \mathrm{NFI}=.97 ; \mathrm{CFI}=.98 ; \mathrm{TLI}=.97$, todos superiores a .95 , que expresan un óptimo ajuste de los datos al modelo teórico. Un índice importante es el RMSEA=.058, el cual resultó inferior a .06 , ratificando la tesis del excelente ajuste de la EAG. El RMSR del modelo fue 0.0471, con un intervalo de confianza obtenido bajo Bootstrap que oscila entre .039 y .051 , el cual contiene el valor medio esperado del RMSR (.042) y se considera un modelo aceptable. Otros índices de ajuste se muestran en la Tabla 6.

\section{Discusión y Conclusiones}

Los hallazgos de este estudio demuestran, en gran medida, características psicométricas apropiadas a una población ecuatoriana entre $19 \mathrm{y}$ 89 años, señalando adecuados indicadores fiabilidad y validez. Los resultados son consistentes con varios estudios realizados con muestras de población general de otros países (Espada, Gonzálvez, Orgilés, Carballo, \& Piqueras, 2012; Luszczynska, Scholz et al., 2005; Scholz, Gutiérrez, Sud, \& Schwarzer, 2002).

Este trabajó mostró también una adecuada consistencia interna, igual o mayor a la reportada en estudios realizados en países latinoamericanos como Chile ( $\alpha=.84$; Cid et al., 2010), Perú $(\alpha=.80)$ y Costa Rica ( $\alpha=.81$; Scholz et al., 2002),México $(\alpha=.86$; Padilla et al., 2006), Argentina $(\alpha=.76$; Brenlla et al., 2010) y comparable con estudios similares en el continente europeo, como en España $(\alpha=.89$; Espada et al., 2012) y las Islas Canarias ( $\alpha=.90$; De las Cuevas \& Peñate, 2015). Esto demuestra que en nuestro contexto, los niveles de confiabilidad superan incluso los presentados en la escala original traducida al español por Bäßler \& Schwarzer (1996) con un alfa de .81 , lo que garantiza la confiabilidad de la escala en nuestro contexto y nos permite confiar en que las mediciones con esta escala no estarán sesgadas por el método seguido (Padilla et al., 2006).

El análisis factorial demostró la unidimensionalidad de la escala, debido a que un solo factor supera el autovalor de $1 \mathrm{y}$ explica el $51.5 \%$ de la prueba; de la misma manera se evidenció estabilidad en los ítems de la prueba, así comoun alto grado de correlación entre los 10 ítems y la suma de los mismos, evidenciándose la mayor (.672) entre los ítems 5 "Gracias a mis cualidades personales yo puedo enfrentar situaciones inesperadas" y 4 "Me siento seguro de poder enfrentar eficazmente situaciones inesperadas". Estudios similares (Cid et al., 2010) señalan una correlación análoga (.633) entre los ítems 6 y 7 , ponderando también la 
unidimensionalidad de la prueba, aunque con valores inferiores (33\%; Brenlla et al., 2010). Estos hallazgos, además de señalar la estabilidad de los ítems, también señalan la solidez de la adaptación a nuestro contexto; lo que resulta importante en palabras deSchwarzer (1999),puesto que destaca la adecuada traducción y redacción de los ítems, generando así correlaciones altas entre ellos.

Por otra parte, las correlaciones positivas entre el bienestar percibido y la autoeficacia, también le otorgan validez a la escala en este estudio; lo que implica que las personas con mayor autoeficacia se perciben además con un nivel alto de bienestar. Esto es particularmente llamativo en el caso de Logro, que se relaciona con el trabajo hacia metas y cumplimiento de tareas; así como Significado, que implica tener un sentido y dirección en la vida. Estos resultados son consistentes con la teoría y con varias investigaciones al respecto. Bandura (1977) por ejemplo, mencionaba que los juicios de autoeficacia se basan en el desempeño de la persona en pro del logro, y esto se comprueba en los estudios de Dijkstra y Ten (2005), quienes señalan que las interpretaciones positivas de autoeficacia contribuyen en gran medida al cumplimiento de metas y objetivos relacionados con el cambio hacia comportamientos saludables. De la misma manera, Lane (2002) determinó en su estudio que el desempeño de boxeadores para la consecución de logros se asocia directamente con la autoeficacia percibida por los mismos.

En referencia al significado de la vida, Lightsey et al. (2014) señalan en su artículo que la autoeficacia general está directamente relacionada con esta dimensión; el equipo explica que mientras mayor sea el significado de vida y conciencia que las personas posean, mayor será el nivel de autoeficacia percibida y esto se verá acompañado de más pensamientos automáticos positivos. De esta manera se explica que el bienestar, tanto en sus dimensiones de Logro como de Significado en la vida, estén íntimamente relacionados con la percepción de autoeficacia; lo que garantiza la validez convergente encontrada en nuestro estudio.

Por otro lado, al analizar la validez divergente se evidencia que la EAG correlaciona de manera negativa con las emociones negativas; este resultado es similar al encontrado por Luszczynska, Scholz et al. (2005), donde mencionan que las creencias fuertes de autoeficacia se relacionan con niveles menores de ansiedad y depresión.

Los estudios también mostraron que existe un ajuste satisfactorio, lo que permite concluir que el modelo se acerca a la explicación de los datos (Espada et al., 2012). La medida del chi cuadrado relativo, en nuestro caso mostró un ajuste excelente al ser menor a 2, aun considerando que algunos autores sugieren que las muestras amplias de los estudios afectan negativamente su resultado y le restan confiabilidad (Scholz et al., 2002); de la misma manera, los demás índices muestran un ajuste óptimo del modelo. Cabe señalar que el valor obtenido del RMSR es considerado aceptable de acuerdo con el criterio de Kelley (1935).

Tal como se ha descrito, los altos niveles de autoeficacia están relacionados con el hecho de que las personas puedan manejar de mejor manera situaciones estresantes o conflictivas (Bandura, 1977), como por ejemplo, que tengan mejor control del dolor en enfermedades crónicas (Schneider et al., 2012; Tewary \& Farber, 2012), o que puedan guiar su conducta al cumplimiento de metas y objetivos (Dijkstra, \& Ten, 2005) y con ello logren una mirada más positiva hacia la vida, con un significado más profundo (Lightsey et al., 2014).

Es así que el hecho de contar con un instrumento de esta magnitud, adaptado al contexto ecuatoriano, implica grandes beneficios para los profesionales de la salud en nuestra sociedad, tanto en el área médica como psicológica. En tal contexto, es necesario conocer el nivel de autoeficacia percibida en las personas, ya que ello dará cuenta de la forma de llevar su tratamiento, e incluso su pronóstico, tal como lo menciona Salvador (2004), una elevada autoeficacia sirve como modelo predictor de adherencia a un tratamiento específico para el control de la enfermedad.

Por ello esta escala de autoeficacia podría implementarse como línea base para establecer una intervención más eficaz y confiable en las personas que requieran un tratamiento, o que tan solo busquen mejorar sus niveles de bienestar (González et al., 2012). De hecho, se debería 
considerar para futuras líneas de investigación, el análisis más detallado de la autoeficacia en poblaciones específicas o en función del sexo y la edad. Con una visión más holística, nos damos cuenta que promover y mejorar los niveles de autoeficacia en las personas, las llevará a la implementacion de intenciones para conseguir sus metas, resolver dificultades que se les presenten (Wieber, Odenthal, \& Gollwitzer, 2010), y incluso generará la motivación necesaria para la realización de una conducta que lleve a una meta y para mantenerse en ella durante largo tiempo (Lewis et al., 2016; Williams \& Rhodes, 2016). Finalmente, las creencias sobre autoeficacia guiarán hacia mejores relaciones sociales en base a la cooperación, conexión, amistad y conducta prosocial (Bandura, Caprara, Barbaranelli, Gerbino, \& Pastorelli, 2003).

En conclusión, la adaptación al español de la Escala de Autoeficacia General para el contexto ecuatoriano, se constituye un instrumento de media eficaz, confiable y válido; brindando la posibilidad de ser aplicada sin perder su capacidad evaluativa. Los análisis realizados confirman la presencia de características psicométricas satisfactorias en esta adaptación, pudiendo utilizar esta escala inclusive a nivel clínico, debido a su alta consistencia interna.

\section{Agradecimientos}

La Escala de Autoeficacia General (EAG) es uno de los primeros trabajos que muestran su adaptación a contextos ecuatorianos, como parte de un proyecto más amplio denominado "Variables asociadas al Bienestar de Personas con y $\sin$ Discapacidad", llevado a cabo gracias al apoyo y financiamiento de la Dirección de Investigación de la Universidad de Cuenca, bajo la dirección del PhD. Mauricio Espinoza.

\section{Referencias}

Acuña-Gurrola, M., \& González-Celis-Rangel, A. (2010). Autoeficacia y red de apoyo en adultos mayores. Journal of Behavior, Health \& Social Issues, 2, 71-81. doi:10.5460/jbhsi.v2.2.26792

Aguado, M., Calvo, D., Dessal, C.,Riechmann, J., González, J., \& Montes, C. 2012. La necesidad de repensar el bienestar humano en un mundo cambiante. Papeles de relaciones ecosociales y cambio global, 119, 49-76. Recuperado de https://dialnet.unirioja.es/ser vlet/articulo?codigo $=4173377$

Bandura, A. (1977). Self-efficacy: Toward a unifying theory of behavioral change. Psychological Review 84, 191-215. doi:10.1037//0033-295x.84.2.191

Bandura, A. (1997). Self-efficacy: The exercise of control. New York, NY: W.H. Freeman and Co.

Bandura, A. (2006). Guide for constructing selfefficacy scales. En F. Pajares, \& T. Urdan (Eds.), Self-efficacy beliefs of adolescents (pp. 307-337). Greenwich, CT: Information Age.

Bandura, A., Caprara, G., Barbaranelli, C., Gerbino, M., \& Pastorelli, C. (2003). Impact of affective self-regulatory efficacy on diverse spheres of functioning. Child Development, 74, 1-14.

Bäßler, J., \& Schwarzer, R. (1996). Evaluación de la autoeficacia: Adaptación española de la escala de autoeficacia general. Ansiedad y Estrés, 2, 1-8.

Brenlla, M., Aranguren, M., Rossaro, M., \& Vázquez, N. (2010). Adaptación para Buenos Aires de la escala de autoeficacia general. Interdisciplinaria, 27, 77-94. Recuperado de http://www.scielo.org.ar/pdf/interd/v27n1/v27 n1a06.pdf

Butler, J., \& Kern, M. (2016). The PERMAProfiler: A brief multidimensional measure of flourishing. International Journal of Wellbeing, 6, 1-48. doi:10.5502/ijw.v6i3.526

Campbell, T., \& Fiske, D. (1959). Convergent and discriminant validation by the multitraitmultimethod matrix. Psychological Bulletin, 56, 81-105. doi: 10.1037/h0046016

Cid, P., Orellana, A., \& Barriga, O. (2010). Validación de la escala de autoeficacia general en Chile. Revista médica de Chile, 138, 551-557. doi:10.4067/s003498872010000500004

Cohen, R., \& Swerdlik, M. (2006). Pruebas y evaluación psicológicas. México, D.F.: MacGraw Hill Interamericana.

Chavarria, J., Stevens, B., Jason, L., \& Ferrari, J., (2012). The effects of self-regulation and selfefficacy on substance use abstinence. 
Alcoholism Treatment Quarterly, 30, 422-432. doi: 10.1080/07347324.2012.718960

De las Cuevas, C., \& Peñate, W. (2015). Validation of the general self-efficacy scale in psychiatric outpatient care. Psicothema, 27, 410-415. doi:10.7334/psicothema2015.56

Del Castillo, A., Guzmán, R., Iglesias, S., \& Reyes, I. (2012). Validez convergente y divergente del instrumento de autoeficacia al tratamiento en diabetes. Psicología Iberoamericana 20, 58-65. Recuperado de http://www.redalyc.org/html/1339/133928816 008/

Dijkstra, A., \& Ten, G. (2005). Ongoing interpretations of accomplishments in smoking cessation: positive and negative selfefficacy interpretations. Addictive Behaviors 30, 219-234.

doi: 10.1016/j.addbeh.2004.05.010

Espada, J., Gonzálvez, M., Orgilés, M., Carballo, J., \& Piqueras J. (2012). Validación de la Escala de Autoeficacia General con adolescentes españoles. Electronic Journal of Research in Educational Psychology, 10(1), 355-370.

González, R., Valle, A., Freire, C., \& Ferradas, M. (2012). Relaciones entre la autoeficacia percibida y el bienestar psicológico en estudiantes universitarios. Revista Mexicana de Psicología, 29, 40-48. Recuperado de http://www.redalyc.org/articulo.oa?id=243030 189004

Grimaldo, M. (2005). Propiedades psicométricas de la escala de autoeficacia general de Baessler \& Schwarzer. Cultura, 19,213-229. Recuperado de http://www.revistacultura.com.pe/portfolioitem/cultura-19/

Hu, L., \& Bentler, P. (1999) Cutoff criteria for fit indexes in covariance structure analysis: Conventional criteria versus new alternatives. Structural Equation Modeling: A Multidisciplinary Journal, 6, 1-55. doi: 10.1080/10705519909540118

Instituto Nacional de Estadísticas y Censos. (2010). Censo de Población y Vivienda. Quito, Ecuador: INEC. Recuperado de https://goo.gl/DqDjcy

International Test Commission. (2016). The ITC guidelines for translating and adapting tests
(Segundaedición).

Recuperado

de: https://www.intestcom.org/

Jöreskog, K., \& Sörbom, D. (2006). LISREL (Versión 8.80) [Software de computación]. Lincolnwood, IL: Scientific Software International.

Kelley, T. (1935). Essential traits of mental life: the purposes and principles underlying the selection and measurement of independent mental factors, together with computational tables. Cambridge: Howard University Press

Klein-Hessling, J., Lohaus, A., \& Ball, J. (2005). Psychological predictors of health-related behaviour in children. Psychology, Health \& Medicine, 10, 31-43. doi: 10.1080/13548500512331315343

Lane, A. (2002). Relationships between performance toward accomplishment and selfefficacy in amateur boxing. Perceptual and motor skills, 94, 1056. Recuperado de https://www.ncbi.nlm.nih.gov/pubmed/12081 268

Lewis, B., Williams, D., Frayeh, A., \& Marcus, B. (2016). Self-efficacy versus perceived enjoyment as predictors of physical activity behavior. Psychology \& Health, 31, 456-469. doi: 10.1080/08870446.2015.1111372

Lightsey, O., Boyraz, G., Ervin, A., Rarey, E., Gharibian, G., \& Maxwell, D. (2014). Generalized self-efficacy, positive cognitions, and negative cognitions as mediators of the relationships between conscientiousness and meaning in life. Canadian Journal of Behavioral Science 46, 436-445. doi: 10.1037/a0034022

Lima-Castro, S., Peña-Contreras, E., CedilloQuizhpe, C., \& Cabrera-Vélez, M. (2016). Adaptación del Perfil PERMA en una muestra ecuatoriana. Manuscrito no publicado.

Lorenzo-Seva, U., \& Ferrando, P. (2006). Factor (Versión 7.0) [Software de computación] España: Universitat Rovira i Virgili

Luszczynska, A., Gutiérrez-Doña, B., \& Schwarzer, R. (2005). General self-efficacy in various domains of human functioning: Evidence from five countries. International Journal of Psychology, 40, 80-89. doi: 10.1080/00207590444000041

Luszczynska, A., Scholz, U., \& Schwarzer, R. (2005).The general self-efficacy scale: 
multicultural validation studies. The Journal of Psychology, 139(5), 439-457.

doi: 10.3200/JRLP.139.5.439-457

Martorell, C., \& Gómez, O. (2010). Enfoque de la evaluación psicológica de la Revista Iberoamericana de Diagnóstico y Evaluación Psicológica (RIDEP). Revista Iberoamericana de Diagnóstico y Evaluación - e Avaliação Psicológica, 30, 35-56. Recuperado de http://www.aidep.org/03_ridep/2_volumen30. html

Molina, M., Raimundi, M., López, C., Cataldi, S., \& Bugallo, L. (2011). Adaptación del perfil de autopercepciones para niños para su uso en la ciudad de buenos aires. Revista Iberoamericana de Diagnóstico y Evaluación - e Avaliação Psicológica, 32, 53-78. Recuperado de http://www.aidep.org/03_ridep/2_volumen32. html

Muñiz, J., Elosua, P., \& Hambleton, R. (2013).Directrices para la traducción y adaptación de los test: segunda edición. Psicothema, 25, 151-157. doi:10.7334/psicothema2013.24

Nickel, D., Spink, K., Andersen, M., \& Knox, K. (2014). Attributions and self-efficacy for physical activity in multiple sclerosis. Psychology, Health \& Medicine, 19, 433-441. doi: 10.1080/13548506.2013.832783

Olivari, C., \& Urra, E. (2007). Autoeficacia y conductas de Salud. Ciencia y Enfermería, 13, 9-15. doi: 10.4067/s0717-95532007000100002

Padilla, J-L., Acosta, B., Gómez, J., Guevara, M., \& González, A. (2006). Propiedades psicométricas de la versión española de la escala de autoeficacia general aplicada en México y España. Revista Mexicana de Psicología, Diciembre-Sin mes, 245252. Recuperado de

http://www.redalyc.org/articulo.oa?id=243020 649010

Reyes, B., Montenegro, E., Knoll, N., \& Schwarzer, R. (2014). Self-efficacy, action control, and social support explain physical activity changes among Costa Rican older adults. Journal of Physical Activity \& Health. 11, 1573-1578. doi: 10.1123/jpah.2013-0175

Reyes-Jarquín, K., \& Hernández-Pozo, M. (2011). Análisis crítico de los estudios que exploran la auto-eficacia y bienestar vinculados al comportamiento saludable. Journal of Behavior, Health \& Social Issues, 3, 5-24. doi: 10.5460/jbhsi.v3.2.29915

Riggio, H., Weiser, D., Valenzuela, A., Lui, P., Montes, R., \& Heuer, J. (2013). Self-efficacy in romantic relationships: Prediction of relationship attitudes and outcomes. The Journal of Social Psychology, 153, 629-650. doi: 10.1080/00224545.2013.801826

Ryan, M. (2008). The antidepressant effects of physical activity: Mediating self-esteem and self-efficacy mechanisms.Psychology \& Health, 23, 279-307. doi: $10.1080 / 14768320601185502$

Salvador, M. (2004). Factores psicológicos y sociales asociados a la adherencia al tratamiento en adolescentes diabéticos tipo 1. Psykhe (Santiago), 13, 21-31. doi: 10.4067/S0718-22282004000100002

Schneider, S., Junghaenel, D., Keefe, F., Schwartz, J., Stone, A., \& Broderick, J. (2012). Individual differences in the day-today variability of pain, fatigue, and well-being in patients with rheumatic disease: Associations with psychological variables. The Journal of the International Association for the Study of Pain, 153, 813-822.

doi: 10.1016/j.pain.2012.01.001

Scholz, U., Gutiérrez, B., Sud, S., \& Schwarzer, R. (2002). Is general self efficacy a universal construct? Psychometric findings from 25 countries. European Journal of Psychological Assessment, 18, 242-251. doi: 10.1027//1015-5759.18.3.242

Schüz, B., Wurm, S., Warner, L., \& Ziegelmann, J. (2012). Self-efficacy and multiple illness representations in older adults: A multilevel approach. Psychology \& Health, 27, 13-29. doi: 10.1080/08870446.2010.541908

Schwarzer, R. (03 de noviembre de 1999).SelfEfficacy Assessment: Recuperado de http://userpage.fu-

berlin.de/ gesund/publicat/ehps_cd/health/wo rld14.htm

Schwarzer, R. (13 de octubre de 2014). The General Self-Efficacy Scale (GSE): Recuperado de http://userpage.fuberlin.de/health/engscal.htm 
Schwarzer, R., Bäßler, J., Kwiatek, P., Schröder, K., \& Zhang, J. (1997). The assessment of optimistic self-beliefs: Comparison of the German, Spanish, and Chinese versions of the General Self-Efficacy Scale. Applied Psychology: An International Review, 46, 6988.

Schwarzer, R., \& Jerusalem, M. (1995). Generalized Self-Efficacy scale. En J. Weinman, S. Wright, \& M. Johnston (Eds.), Measures in health psychology: A user's portfolio. Causal and control beliefs (pp. 35-37). Windsor, UK: Nfer-Nelson.

Schwarzer, R., \& Warner, L. (2013). Perceived self-efficacy and its relationship to resilience. En S. Prince-Embury \& D. Saklofske (Eds.), Resilience in children, adolescents, and adults: Translating research into practice (pp. 139-150). doi: 10.1007/978-1-4614-4939-3_10

Seligman, M. (2012). Flourish: A visionary new understanding of happiness and well-being. New York, NY: The Free Press.

Singh, A., Shukla, A., \& Singh, P. (2010). Perceived Self Efficacy and Mental Health among Elderly. Delhi Psychiatry Journal, 13, 314-321. Recuperado de http://medind.nic.in/daa/t10/i2/daat10i2p314.p df

Tewary, S., \& Farber, N. (2012). Examining interactions of marriage and self-efficacy beliefs of individuals with rheumatoid arthritis: A conceptual framework. Journal of Human Behavior in the Social Environment, 22, 707-717. doi:10.1080/10911359.2012.692561

Wieber, F., Odenthal, G., \& Gollwitzer, P. (2010). Self-efficacy feelings moderate implementation intentions effects. Self and Identity, 9, 177-194. doi: 10.1080/15298860902860333

Williams, D., \& Rhodes, R. (2016). The confounded self-efficacy construct: conceptual analysis and recommendations for future research. Health Psychology Review, 10, 113-128. doi: 10.1080/17437199.2014.941998 